PROCEEDINGS OF THE

AMERICAN MATHEMATICAL SOCIETY

Volume 135, Number 9, September 2007, Pages 3013-3019

S 0002-9939(07)08837-5

Article electronically published on May 14, 2007

\title{
HAMILTON'S GRADIENT ESTIMATE FOR THE HEAT KERNEL ON COMPLETE MANIFOLDS
}

\author{
BRETT L. KOTSCHWAR
}

(Communicated by Richard A. Wentworth)

\begin{abstract}
In this paper we extend a gradient estimate of R. Hamilton for positive solutions to the heat equation on closed manifolds to bounded positive solutions on complete, non-compact manifolds with $R c \geq-K g$. We accomplish this extension via a maximum principle of L. Karp and P. Li and a Berstein-type estimate on the gradient of the solution. An application of our result, together with the bounds of P. Li and S.T. Yau, yields an estimate on the gradient of the heat kernel for complete manifolds with non-negative Ricci curvature that is sharp in the order of $t$ for the heat kernel on $\mathbb{R}^{n}$.
\end{abstract}

\section{INTRODUCTION}

In $[\mathrm{H}$, Richard Hamilton established the following estimate on the gradient of the logarithm of a positive solution to the heat equation.

Theorem (Hamilton). Suppose $\left(\mathcal{M}^{n}, g\right)$ is a closed Riemannian manifold and $u$ a positive solution to the heat equation on $\mathcal{M}^{n}$. If $M>0$ and $K \geq 0$ are constants such that $R c \geq-K g$ and $u(x, t) \leq M$, then for all $x \in \mathcal{M}^{n}$ and $t>0$, one has

$$
t|\nabla \log (u)|^{2} \leq(1+2 K t) \log (M / u) .
$$

In this paper, we provide a proof that Hamilton's theorem also holds for complete, non-compact manifolds with Ricci curvature bounded below. Under the additional restriction of non-negative Ricci curvature, we then obtain, via the wellknown bounds of Li and Yau [LY], the following estimate on the heat kernel. (Recall that on a complete, non-compact manifold, the heat kernel may be defined as the smallest positive fundamental solution to the heat equation.)

Theorem 1. Suppose $\mathcal{M}^{n}$ is a complete, non-compact manifold with $R c \geq 0$, and $H$ its heat kernel. Then, for all $\delta>0$, there exists a constant $C=C(n, \delta)$ such that

$$
|\nabla \log (H(x, y, t))|^{2} \leq \frac{2}{t}\left(C+\frac{d(x, y)^{2}}{(4-\delta) t}\right)
$$

for all $x, y$ in $\mathcal{M}^{n}$ and $t>0$.

Theorem 1 is sharp in the order of $t$ for the heat kernel on $\mathbb{R}^{n}$ and should be compared to the recent estimate of Souplet and Zhang in $[\mathrm{SZ}]$ which applies to the

Received by the editors March 13, 2006 and, in revised form, June 23, 2006.

2000 Mathematics Subject Classification. Primary 58J35; Secondary 35K05.

(C) 2007 American Mathematical Society 
heat kernel on manifolds with $R c(g) \geq-K g$. In the special case $K=0$, inequality (21) is comparable to their estimate at scales $d^{2}(x, y) \leq c t$ and offers an improvement at scales $t<<d^{2}(x, y)$.

Additionally, such an estimate is required to prove that the integrand in the entropy functional $\mathcal{W}$ for the linear heat equation (cf. [N2]) is pointwise nonpositive for the fundamental solution to the heat equation. The proof that the integrand in Perelman's $\mathcal{W}$-functional is non-positive for fundamental solutions to the conjugate heat equation seems also to require a non-linear analog of this result (see $[\mathbf{N}]$ ). Perhaps the approach detailed here could serve as a model for the proof of such an estimate.

In Section 2, we obtain a Bernstein-type estimate for bounded solutions to the heat equation which affords pointwise control on the product of the squared norm of the gradient by the elapsed time. The estimate is similar in form to those found by W.-X. Shi $[\mathrm{S}]$ in the Ricci Flow setting for derivatives of curvature. Such estimates have found considerable service in that setting and continue to have importance in work towards the verification of the claims of Perelman.

Note added in proof. The recent work of Ni [N2] and Zhang [Z] (see also KuangZhang [KZ]) contains estimates very similar to our global estimate (Theorem 6) and its application to the heat kernel, as well as analogues for solutions coupled to a metric evolving by the Ricci flow, and their applications to Perelman's differential Harnack estimate $[\mathrm{P}$.

\section{A Bernstein-type estimate}

Henceforth we shall assume that $\left(\mathcal{M}^{n}, g\right)$ is a smooth, complete, non-compact Riemannian manifold with Ricci curvature uniformly bounded below by $-K$, and for this section, suppose $u$ is a smooth solution to the heat equation on some open $U \subset \mathcal{M}^{n}$ for $0 \leq t \leq T \leq \infty$, satisfying $|u| \leq M$. Our aim is to establish a preliminary estimate on $|\nabla u|^{2}$ so that the maximum principle of Ni and Tam [NT] may be applied to the quantity of interest in Hamilton's gradient estimate.

To do this, we employ a technique of W.-X. Shi $[\mathrm{S}]$ from the estimation of derivatives of curvature under the Ricci Flow (see also the treatment in the forthcoming book [CLN] $)$, and define $F(x, t)=\left(4 M^{2}+u^{2}\right)|\nabla u|^{2}$ for $t>0$. The evolution of $F$ then possesses an advantageous $-F^{2}$ term, as we see below.

Lemma 2. There exist positive constants $C_{1}$ and $C_{2}$ such that

$$
\frac{\partial F}{\partial t} \leq \Delta F+C_{1} K F-\frac{C_{2}}{M^{4}} F^{2}
$$

Proof. We have

$$
\left(\frac{\partial}{\partial t}-\Delta\right)|\nabla u|^{2}=-2|\nabla \nabla u|^{2}-2 R c(\nabla u, \nabla u) \leq-2|\nabla \nabla u|^{2}+2 K|\nabla u|^{2},
$$

and

$$
\left(\frac{\partial}{\partial t}-\Delta\right) u^{2}=-2|\nabla u|^{2}
$$


So

$$
\begin{gathered}
\left(\frac{\partial}{\partial t}-\Delta\right) F \leq\left(4 M^{2}+u^{2}\right)\left(2 K|\nabla u|^{2}-2|\nabla \nabla u|^{2}\right)-2|\nabla u|^{4} \\
-8 u(\nabla \nabla u)(\nabla u, \nabla u) \\
\leq-10 u^{2}|\nabla \nabla u|^{2}+10 M^{2} K|\nabla u|^{2}-2|\nabla u|^{4} \\
-8 u(\nabla \nabla u)(\nabla u, \nabla u) .
\end{gathered}
$$

Since

$$
8|u||\nabla \nabla u||\nabla u|^{2} \leq 10 u^{2}|\nabla \nabla u|^{2}+\frac{8}{5}|\nabla u|^{4},
$$

and $4 M^{2}|\nabla u|^{2} \leq F \leq 5 M^{2}|\nabla u|^{2}$, we find

$$
\begin{aligned}
\left(\frac{\partial}{\partial t}-\Delta\right) F & \leq 10 M^{2} K|\nabla u|^{2}-\frac{2}{5}|\nabla u|^{4} \\
& \leq \frac{5}{2} K F-\frac{2}{625 M^{4}} F^{2}
\end{aligned}
$$

as claimed.

Now, as in [LY], for any $p \in \mathcal{M}^{n}$ and $R>0$ we may find a cut-off function $\eta(x)=$ $\eta_{p, R}(x)$ equal to 1 on $B_{p}(R)$ and supported in $B_{p}(2 R)$ satisfying the conditions

$$
|\nabla \eta|^{2} \leq \frac{C_{3}}{R^{2}} \eta
$$

and

$$
\Delta \eta \geq-\frac{C_{3}}{R^{2}}(1+R \sqrt{K})
$$

for some $C_{3}=C_{3}(n)>0$. Strictly speaking, the above estimates need only hold away from the cut-locus of $p$, however, for the purposes of applying the maximum principle to $\eta F$, the well-known argument of Calabi [C] allows us to assume that they hold everywhere.

The main result of this section is the following local estimate:

Theorem 3. Suppose $u$ is a smooth solution to the heat equation satisfying $|u| \leq M$ on $B_{p}(2 R) \times[0, T]$ for some $p \in \mathcal{M}^{n}$ and $M, R, T>0$. Then there exists a constant $C_{4}=C_{4}(K)$ such that

$$
t|\nabla u|^{2} \leq C_{4} M^{2}\left(1+T\left(1+\frac{1}{R^{2}}\right)\right)
$$

holds on $B_{p}(R) \times[0, T]$. 
Proof. Define $F$ as in Lemma 2 . On $\operatorname{supp}(\eta) \times[0, T]$, we have, by Lemma 2 and equations (4) and (5),

$$
\begin{aligned}
\left(\frac{\partial}{\partial t}-\Delta\right)(t \eta F)= & \eta F+t \eta\left(\frac{\partial}{\partial t}-\Delta\right) F-t F \Delta \eta-2 t\langle\nabla \eta, \nabla F\rangle \\
= & \left(\eta+2 t \frac{|\nabla \eta|^{2}}{\eta}-t \Delta \eta\right) F+t \eta\left(\frac{\partial}{\partial t}-\Delta\right) F \\
& \quad-2\left\langle\nabla(t \eta F), \frac{\nabla \eta}{\eta}\right\rangle \\
\leq & \left(\left(1+C_{1} K t\right) \eta+3 t \frac{C_{3}}{R^{2}}(1+R \sqrt{K})\right) F-\frac{C_{2}}{M^{4}} t \eta F^{2} \\
& \quad-2\left\langle\nabla(t \eta F), \frac{\nabla \eta}{\eta}\right\rangle .
\end{aligned}
$$

If $\eta F$ is not identically zero (i.e., if $u$ is not constant on $\operatorname{supp}(\eta)$ ), then $t \eta F$ attains a positive maximum at $\left(x_{0}, t_{0}\right) \in \mathcal{M}^{n} \times(0, T]$.

At this point,

$$
\nabla(t \eta F)=0
$$

and

$$
\left(\frac{\partial}{\partial t}-\Delta\right)(t \eta F) \geq 0
$$

so

$$
\frac{C_{2}}{M^{4}} t_{0} \eta F^{2} \leq\left(1+C_{1} K T+3 T \frac{C_{3}}{R^{2}}(1+R \sqrt{K})\right) F .
$$

Consequently, for any $(x, t) \in B_{p}(R) \times[0, T]$,

$$
\begin{aligned}
t F(x, t) & =t F(x, t) \eta(x) \\
& \leq t_{0} F\left(x_{0}, t_{0}\right) \eta\left(x_{0}\right) \\
& \leq \frac{M^{4}}{C_{2}}\left(1+C_{1} K T+3 T \frac{C_{3}}{R^{2}}(1+R \sqrt{K})\right) .
\end{aligned}
$$

But $|\nabla u|^{2} \leq\left(1 / 4 M^{2}\right) F$, and the claim follows.

Remark 4. If $R c \geq 0$, the above proof shows

$$
t|\nabla u|^{2} \leq C M^{2}\left(1+\frac{T}{R^{2}}\right)
$$

on $B_{p}(R) \times[0, T]$.

Sending $R \rightarrow \infty$ in the penultimate line of the above proof, we at once obtain

Corollary 5. Suppose the solution u exists on all of $\mathcal{M}^{n} \times[0, T]$. Then there exists a constant $C_{5}$ such that

$$
t|\nabla u|^{2} \leq C_{5} M^{2}(1+K T)
$$

on $\mathcal{M}^{n} \times[0, T]$. 


\section{Proof of the MAIN THEOREM}

Next, using the estimate of the previous section, we apply a maximum principle due originally to Karp and Li whose statement we found (in more generalized form) in a paper of $\mathrm{Ni}$ and Tam. The statement of their theorem in our (stationary metric) case is as follows. Here $f_{+}(x, t):=\max \{f(x, t), 0\}$.

Theorem (Karp-Li [KL] and Ni-Tam [NT, 1.2). Suppose $\left(\mathcal{M}^{n}, g\right)$ is a complete Riemannian manifold and $f(x, t)$ a smooth function on $\mathcal{M}^{n} \times[0, T]$ such that $\left(\frac{\partial}{\partial t}-\Delta\right) f(x, t) \leq 0$ whenever $f(x, t) \leq 0$. Assume that

$$
\int_{0}^{T} \int_{\mathcal{M}^{n}} e^{-a r^{2}(x)} f_{+}^{2}(x, s) d V d s<\infty
$$

for some $a>0$, where $r(x)$ is the distance to $x$ from some fixed $p \in \mathcal{M}^{n}$. If $f(x, 0) \leq 0$ for all $x \in \mathcal{M}^{n}$, then $f(x, t) \leq 0$ for all $(x, t) \in \mathcal{M}^{n} \times[0, T]$.

Hamilton's Theorem in the complete case reads as

Theorem 6. Suppose $\left(\mathcal{M}^{n}, g\right)$ is a complete manifold with $R c \geq-K g$ for some $K \geq 0$. If $0<u(x, t) \leq M$ is a solution to the heat equation on $\mathcal{M}^{n} \times[0, T]$ for $0<T \leq \infty$, then

$$
t|\nabla \log u|^{2} \leq(1+2 K t) \log (M / u) .
$$

Proof. Defining $u_{\epsilon}=u+\epsilon$ for $\epsilon>0$, we obtain a solution satisfying $\epsilon<u_{\epsilon} \leq$ $M+\epsilon:=M_{\epsilon}$. Once the estimate has been proved for $u_{\epsilon}$, the theorem will follow by letting $\epsilon \rightarrow 0$.

As in $[\mathrm{H}]$, the function

$$
P(x, t):=\varphi(t) \frac{\left|\nabla u_{\epsilon}\right|^{2}}{u_{\epsilon}}-u_{\epsilon} \log \left(\frac{M_{\epsilon}}{u_{\epsilon}}\right),
$$

where $\varphi(t):=t /(1+2 K t)$, satisfies $\left(\frac{\partial}{\partial t}-\Delta\right) P(x, t) \leq 0$ and

$$
P(x, 0)=-u_{\epsilon} \log \left(M_{\epsilon} / u_{\epsilon}\right) \leq 0 .
$$

By our assumptions on $u_{\epsilon}$, we also have

$$
P_{+}(x, t) \leq \frac{1}{\epsilon} t\left|\nabla u_{\epsilon}\right|^{2}
$$

Thus using equation (7), for any $p \in \mathcal{M}^{n}$, and $R>0$, we have

$$
\begin{aligned}
\int_{0}^{T} \int_{B_{p}(R)} e^{-r^{2}(x)} P_{+}^{2}(x, t) d V & d t \leq \frac{1}{\epsilon^{2}} \int_{0}^{T} \int_{B_{p}(R)} e^{-r^{2}(x)}\left(t\left|\nabla u_{\epsilon}\right|^{2}\right)^{2} d V d t \\
\leq & \frac{C_{5}^{2} M_{\epsilon}^{4}}{\epsilon^{2}}(1+K T)^{2} \int_{0}^{T} \int_{\mathcal{M}^{n}} e^{-r^{2}(x)} d V d t
\end{aligned}
$$

Since we assume that $R c \geq-K g$, it follows from the Bishop volume comparison theorem that the rightmost integral in the above inequality is finite. 
Hence,

$$
\begin{aligned}
\int_{0}^{T} \int_{\mathcal{M}^{n}} e^{-r^{2}(x)} P_{+}^{2}(x, t) d V d t & \leq \liminf _{R \rightarrow \infty} \int_{0}^{T} \int_{B_{p}(R)} e^{-r^{2}(x)} P_{+}^{2}(x, t) d V d t \\
& <\infty,
\end{aligned}
$$

and we conclude that $P(x, t) \leq 0$ for all $t \leq T$.

Proof of Theorem 1. Let $H(x, y, t)$ denote the heat kernel of $\left(\mathcal{M}^{n}, g\right)$. For any $t>0$ and $y \in \mathcal{M}^{n}$, set $u(x, s):=H(x, y, s+t / 2)$. Then $u$ is a smooth, positive solution to the heat equation on $[0, \infty)$. By Corollary 3.1 and Theorem 4.1 of $[\mathrm{LY}$, for all $\delta>0$, there is a constant $C_{6}=C_{6}(\delta)>0$ such that $u$ satisfies

$$
C_{6}^{-1} V(\sqrt{s+t / 2})^{-1} e^{\frac{-d^{2}(x, y)}{(4-\delta)(s+t / 2)}} \leq u(x, s) \leq C_{6} V(\sqrt{s+t / 2})^{-1}
$$

for all $x, y \in \mathcal{M}^{n}$, and $s \geq 0$, where $V(\sqrt{s+t / 2}):=\operatorname{Vol}\left(B_{y}(\sqrt{t+s / 2})\right.$.

Defining $M=C_{6} V(\sqrt{t / 2})^{-1}$, the left inequality in (9) implies $u \leq M$ for all $x$ and $s$. Moreover, since we assume $R c \geq 0$, there exists a positive constant $C_{7}=C_{7}(n)$ such that for all $0 \leq s \leq t / 2$

$$
V(\sqrt{t / 2+s}) \leq V(\sqrt{t}) \leq C_{7} V(\sqrt{t / 2}) .
$$

Thus, by the right-hand inequality in (9) and Theorem 6 , we have

$$
s|\nabla \log u|^{2} \leq \log \left(\frac{M}{u}\right) \leq\left(\log \left(C_{6}^{2} C_{7}\right)+\frac{d^{2}(x, y)}{(4-\delta)(s+t / 2)}\right)
$$

on $\mathcal{M}^{n} \times[0, t / 2]$.

Setting $C=\log \left(C_{6}^{2}(\delta) C_{7}(n)\right)$ and evaluating at $s=t / 2$, we conclude that

$$
(t / 2)|\nabla \log H|^{2}(x, y, t)=(t / 2)|\nabla u|^{2}(x, t / 2) \leq\left(C+\frac{d^{2}(x, y)}{(4-\delta) t}\right)
$$

for all $x, y \in \mathcal{M}^{n}$ and $t>0$.

\section{REFERENCES}

[C] Calabi, E. An extension of E. Hopf's maximum principle with an application to Riemannian geometry. Duke Math. J. 25 (1957) 45-56. MR0092069(19:1056e)

[CLN] Chow Bennett; Lu, Peng; Ni, Lei. Hamilton's Ricci Flow. Lectures in Contemporary Mathematics, Science Press, and Graduate Studies in Mathematics, Vol. 77, American Mathematical Society, Providence, RI, 2006.

[H] Hamilton, Richard S. A matrix Harnack estimate for the heat equation. Comm. Anal. Geom. 1 (1993), no.1, 113-126. MR.1230276 (94g:58215)

[KL] Karp, L. and Li, P. The heat equation on complete riemannian manifolds. Unpublished notes, 1982.

[KZ] Kuang, S. and Zhang, Q. S. A gradient estimate for all positive solutions of the conjugate heat equation under Ricci flow. arXiv:math/0611298

[LY] Li, Peter and Yau, S.-T. On the parabolic kernel of the Schrödinger operator. Acta Math. 156 (1986), no. 3-4, 153-201. MR0834612 (87f:58156)

[N] Ni, Lei. A note on Perelman's LYH inequality. arXiv:math.DG/0602337

[N2] Ni, Lei. The entropy formula for linear heat equation. J. Geom Anal. 14 (2004), no. 1, 87-100. MR 2030576 (2004m:53118a)

[NT] Ni, Lei and Tam, L. F. Kähler-Ricci flow and the Poincarè-Lelong equation. Comm. Anal. Geom 12 (2004), no. 1-2, 111-141. MR2074873(2005f:53108)

[P] Perelman, Grisha. The entropy formula for the Ricci flow and its Geometric Applications. arXiv:math.DG/0211159 
[S] Shi, Wan-Xiong Deforming the metric on complete Riemannian manifolds. J. Differential Geom. 30 (1989), no. 1, 223-301. MR.1001277 (90i:58202)

[SZ] Souplet, P. and Zhang, Q.S. Sharp gradient estimate and Yau's Liouville theorem for the heat equation on non-compact manifolds, arXiv:math.DG/0502079

[Z] Zhang, Q. S. Some gradient estimates for the heat equation and for an equation by Perelman. Int. Math. Res. Not. 2006, Art. 1D 92314, 39 pp. MR2250008 (2007f:35116)

Department of Mathematics, University of California, San Diego, California 92110

E-mail address: bkotschw@math.ucsd.edu 